\title{
A nudge towards better lumbar puncture practice
}

\author{
Authors: Christian Holland, ${ }^{\mathrm{A}}$ Evan C Edmond, ${ }^{\mathrm{B}}$ Catherine Moore, ${ }^{\mathrm{A}}$ Vanessa Tobert, ${ }^{\mathrm{C}}$ Johannes C Klein ${ }^{\mathrm{D}}$ and \\ Martin R Turner ${ }^{\mathrm{D}}$
}

\section{Background}

Despite a body of evidence demonstrating reduced incidence of post-lumbar puncture headache associated with pencil-point (vs bevelled-edge) needles, their use remains variable in the UK.

\section{Methods}

A multimodal longitudinal intervention was performed over a 12-month period at a tertiary neurology referral centre. In addition to simulation training using pencil-point needles and an electronic documentation pro forma, a change in the default needles presented in clinical environments was performed.

\section{Results}

Prior to the intervention, pencil-point needle usage was minimal. Documentation significantly improved throughout the intervention period. Simulation training interventions only resulted in transient, moderate improvements in pencilpoint needle usage. However, changing the default produced a marked increase in use that was sustained. No significant changes in operator success rate were found.

Conclusions

In the context of wider literature on the power of default options in driving behavioural choices, changing defaults may be an effective, inexpensive and acceptable intervention to improve lumbar puncture practice.

KEYWORDS: Neurology, quality improvement, lumbar puncture

DOI: 10.7861/clinmed.2020-0201

\section{Introduction}

Headache is a historically known complication of lumbar puncture (LP). In 1899, August Bier and his assistant August Hildebrandt performed LPs on each other attempting to reproduce complications from spinal anaesthesia in humans. ${ }^{1}$ Both men developed severe headaches beginning the following day. For a further 8 days, Bier

Authors: ${ }^{\text {A }}$ medical student, University of Oxford, Oxford, UK; ${ }^{B}$ clinical research fellow, University of Oxford, Oxford, UK and Oxford University Hospitals NHS Foundation Trust, Oxford, UK; ${ }^{C}$ core medical trainee, Oxford University Hospitals NHS Foundation Trust, Oxford, UK; D neurology consultant, University of Oxford, Oxford, UK and Oxford University Hospitals NHS Foundation Trust, Oxford, UK was forced to lie flat as rising provoked severe pain. This classical description remains very relevant to modern clinical practice. Postdural-puncture headache (PDPH) commonly causes morbidity, in serious cases requiring hospital admission or epidural blood patch. ${ }^{2-4}$ One key modifier of these risks is spinal needle design.

A wide variety of designs have been used historically. ${ }^{5}$ The bevelled tip of the conventional Quincke needle readily cuts through tough ligamentous structures and the dura mater. With electron microscopy, the dural defect left by this needle is seen as a crescent-shaped flap with cleanly-cut borders. ${ }^{6}$ This may allow persistent cerebrospinal fluid (CSF) leak and intracranial hypotension to develop, leading to headache. To address this problem, needles with blunter 'pencil-point' or 'atraumatic' tips were designed with the aim of dividing rather than cutting dural fibres (Fig 1). The Greene, Whitacre and Sprotte needles were introduced in the 1920s, 1950s, and 1980s, respectively.

\section{Quincke}

\section{Sprotte}
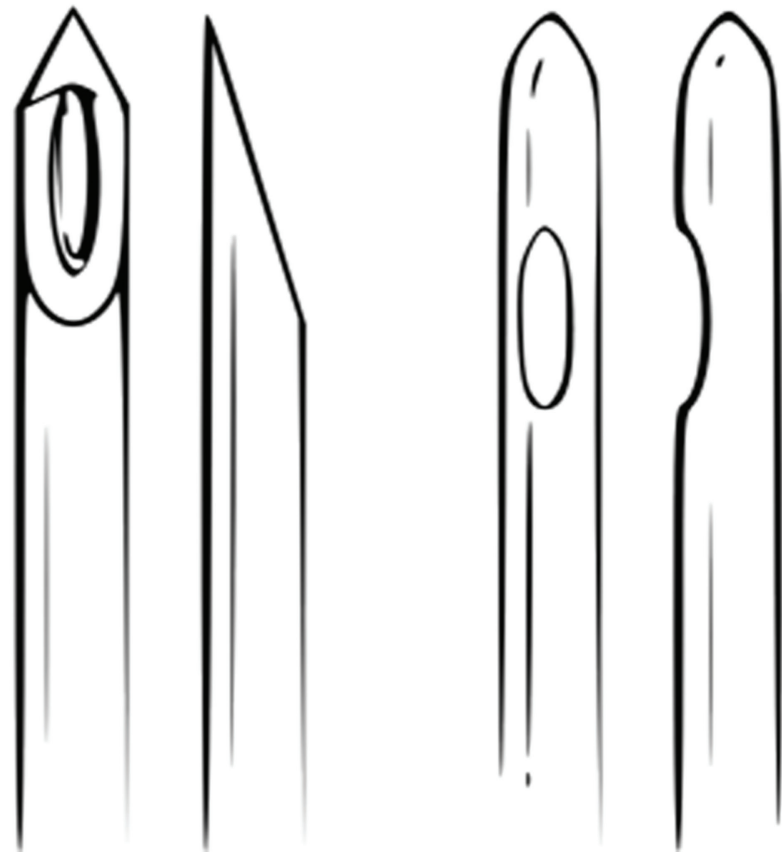

Fig 1. Examples of different needles: sharp tip (Quincke) and blunter 'pencil-point' or 'atraumatic' tip (Sprotte). 
These needles form irregular defects with ragged borders and a smaller cross-sectional area on the arachnoid surface. ${ }^{6}$ Mechanically, these defects may be less likely to allow persistent CSF leak. The greater tissue destruction at the ragged edges has also been suggested to provoke inflammation, facilitating closure. Precise mechanism notwithstanding, if needle design mitigates headache risk, a clinician's choice of needle becomes an important determinant of LP outcomes.

Meta-analyses suggest that larger diameter needles and cutting needle tips increase headache risk. ${ }^{7,8}$ The difference is clinically meaningful, with PDPH incidence, intravenous fluid or analgesia requirement, and blood patch all reduced by at least $50 \%$ with pencil-point needles. LP success rate is unchanged. Where equivalent, replacing cutting needles with pencil-points would benefit patients and save healthcare resources. ${ }^{8,9}$

Adoption of pencil-point needles as the standard of care is widespread in the original context of their development - spinal anaesthesia. ${ }^{10}$ However, in other clinical settings where LP is performed (acute medicine, neurology and neurosurgery) cutting needles remain in common clinical use despite various interventions. ${ }^{11}$ This study reports a multimodal intervention where, in addition to standard interventions, the effect of replacing the default stocked needle in the ward environment was assessed.

\section{Methods}

The clinical population was ambulatory outpatients who had diagnostic LP, generally prior to neurological diagnosis. This group did not include inpatient admissions to acute medicine. Patients having large-volume CSF drainage for idiopathic intracranial hypertension were also excluded. Electronic records were reviewed for diagnostic LPs at the neurology investigations unit (NIU), Oxford University Hospitals NHS Foundation Trust, from October 2017 to December 2018. LPs from October 2016 were a pre-intervention reference. Pencil-point needle usage was recorded in addition to a documentation quality score based on local and national clinical guidelines. One point for recording each of (to a maximum of seven) procedure indication, platelets/ clotting checked, causes of intracranial pressure reviewed,

informed consent obtained, local anaesthetic administration, CSF appearance and tests requested.

The intervention comprises three areas.

$>$ Electronic pro forma for documentation (introduced September 2017) recording pre-procedure safety checks, consent, patient positioning, type of needle used and investigations requested.

$>$ Simulation training sessions for clinicians performing LPs using a training model and pencil-point needles. Sessions took place in September 2017, February 2018 and July 2018.

> Changing the default needle. In September 2018, the 22G Quincke needles stocked by default in trolleys and cupboards on the NIU were replaced with 22G Sprotte needles. Quincke needles remained accessible in ward stores if specifically indicated for therapeutic CSF drainage.

\section{Results}

Between October 2017 and December 2018, 210 LPs were recorded. Documentation score improved with the pro forma and training interventions. However, prior to the change in default needle, pencilpoint needle usage was variable. In the 3 months after each training session, transient increases in atraumatic needle usage were seen. After the 1st intervention (proforma and simulation training) in September 2017, mean pencil-point needle usage October-December 2017 was $37 \%$. Following a 2 nd intervention (simulation training) in February 2018, mean pencil-point needle usage was 23\%. However, usage returned to near baseline following these increases (Fig 2).
Fig 2. Monthly atraumatic needle usage and mean documentation score.

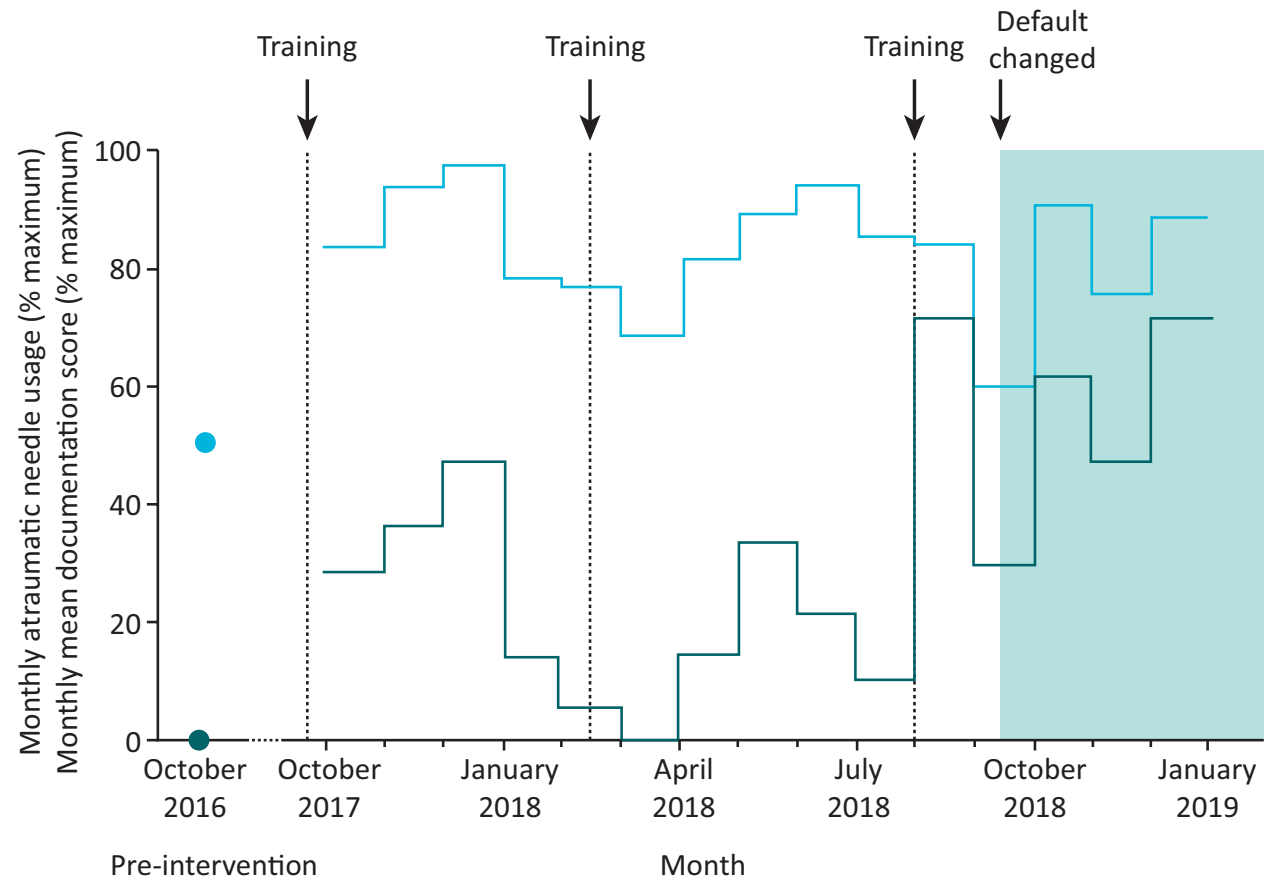

Pre-intervention
Documentation

Atraumatic 
On changing the default needle available on the ward in September 2018, a larger and sustained increase in mean pencilpoint needle usage over the subsequent 3 months was seen (60.1\%). Clinician success rate did not differ throughout the study period with cutting vs pencil-point needles ( $48 \%$ vs $46 \% ; p=0.4$ ).

\section{Discussion}

Our multimodal intervention increased pencil-point needle usage in this centre, illustrating the importance of systemic factors in clinical medicine. Training and pro forma interventions have previously been characterised by transient effects, diluted by staff turnover and fatigue. In this study, the default needle in trolleys and stock cupboards was changed to pencil-point, which was associated with a more sustained change in needle usage.

Behavioural economics describes the marked influence on human decision-making of a 'choice environment' composed of default settings, presentation of options and a range of other subtle cues. ${ }^{12}$ No choice environment is neutral, and existing structures may favour decisions that cause undesirable outcomes. 'Choice architecture' is the design of small alterations, described as 'nudges', that avoid undue restriction, while promoting decisions associated with a desired outcome. A range of ethical and practical caveats accompany these design choices, and interventions based on behavioural theories require a robust evidence base to justify implementation.

Changing defaults is a well-established method to drive behavioural change more widely in medicine. ${ }^{13}$ Opt-out systems for organ donation result in substantially more organs available for transplant. ${ }^{14}$ Changing defaults in electronic prescribing systems promotes cheaper generic prescribing. ${ }^{15}$ Far fewer urinary tract infections occur when indwelling urinary catheters are routinely removed after a certain time. ${ }^{16}$ More widely, people opt in to receive email marketing, change their energy supplier and open retirement savings at rates strongly determined by default options. ${ }^{17-19}$

By following up three different intervention styles (pro forma, training and choice architecture), we are able to differentiate the impact of these interventions and observe their impact over time. Through capture of all LPs done in a single unit by a range of clinical staff of similar background in the same environment, we reduce ecological effects and site-specific confounders. Unforeseen negative effects of the intervention were actively investigated, anonymous clinician feedback was solicited, and success rate with needle type was assessed. However, the scope of this study was too limited to detect significant differences in readmission and blood patch. With regards to headache, accurately determining its rate from clinical records is challenging. Follow-up across the entire time range over which headache can occur was considered outside the scope of this study. Another limitation is the difficulty in inferring changes in clinician choice from clinical documentation; individual procedures were not monitored.

\section{Conclusion}

This study resulted in sustained improvement in lumbar puncture practice at this centre. Of the interventions performed, choice architecture was an effective, inexpensive and acceptable approach. In appropriate clinical environments and in the absence of mandated needle choice, it offers significant improvement over previously described interventions to improve LP.

\section{References}

1 Bier A. [Experiments on the cocainization of the spinal cord]. Dtsch Z Für Chir 1899;51:361-9.

2 Greene $\mathrm{H}$. Lumbar puncture and the prevention of postpuncture headache. J Am Med Assoc 1936;86:391-2.

3 Lavi R, Yarnitsky D, Rowe JM et al. Standard vs atraumatic Whitacre needle for diagnostic lumbar puncture: a randomized trial. Neurology 2006;67:1492-4.

4 Turnbull D, Shepherd D. Post-dural puncture headache: pathogenesis prevention and treatment. Br J Anaesth 2003;91:718-29.

5 Calthorpe N. The history of spinal needles: getting to the point. Anaesthesia 2004:59:1231-41.

6 Reina MA, Puigdellívol-Sánchez A, Gatt SP et al. Electron microscopy of dural and arachnoid disruptions after subarachnoid block. Reg Anesth Pain Med 2017:42:709-18.

7 Halpern S, Preston R. Postdural puncture headache and spinal needle design Metaanalyses. Anesthesiology 1994;81:1376-83.

8 Rochwerg B, Siemieniuk RAC, Agoritsas T et al. Atraumatic (pencilpoint) versus conventional needles for lumbar puncture: a clinical practice guideline. BMJ 2018;361:k1920.

9 Dakka Y, Warra N, Albadareen R, Jankowski M, Silver B. Headache rate and cost of care following lumbar puncture at a single tertiary care hospital. Neurology 2011;77:71-4.

10 Lybecker H, Møller JT, May O, Nielsen HK. Incidence and prediction of postdural puncture headache A prospective study of 1021 spinal anesthesias. Anesth Analg 1990;70:389-94.

11 Davis A, Dobson R, Kaninia S, Giovannoni G, Schmierer K. Atraumatic needles for lumbar puncture: why haven't neurologists changed? Pract Neurol 2016;16:18-22.

12 Shafir E. The behavioral foundations of public policy. Princeton University Press, 2013.

13 Halpern SD, Ubel PA, Asch DA. Harnessing the power of default options to improve health care. N Engl J Med 2007;357:1340-4.

14 Davidai S, Gilovich T, Ross LD. The meaning of default options for potential organ donors. Proc Natl Acad Sci 2012;109:15201-5.

15 Patel MS, Day SC, Halpern SD et al. Generic medication prescription rates after health System-Wide redesign of default options within the electronic health record. JAMA Intern Med 2016;176:847-8.

16 Cornia PB, Amory JK, Fraser S, Saint S, Lipsky BA. Computer-based order entry decreases duration of indwelling urinary catheterization in hospitalized patients. Am J Med 2003;114:404-7.

17 Johnson EJ, Bellman S, Lohse GL. Defaults framing and privacy: Why opting in-opting out. Mark Lett 2002;13:5-15.

18 Pichert D, Katsikopoulos KV. Green defaults: Information presentation and pro-environmental behaviour. J Environ Psychol 2008;28:63-73.

19 McKenzie CR, Liersch MJ, Finkelstein SR. Recommendations implicit in policy defaults. Psychol Sci 2006;17:414-20.

Address for correspondence: Prof Martin R Turner, Nuffield Department of Clinical Neuroscience, Level 6 West Wing, John Radcliffe Hospital, Headley Way, Oxford OX3 9DU, UK. Email: martin.turner@ndcn.ox.ac.uk 